\title{
BMJ Open Lived experiences of healthcare workers on the front line during the COVID-19 pandemic: a qualitative interview study
}

\author{
Kate Grailey (D) , ${ }^{1}$ Adam Lound, ${ }^{2}$ Stephen Brett (i) ${ }^{3}$
}

To cite: Grailey K, Lound A, Brett S. Lived experiences of healthcare workers on the front line during the COVID-19 pandemic: a qualitative interview study. BMJ Open 2021;11:e053680. doi:10.1136/ bmjopen-2021-053680

- Prepublication history and additional supplemental material for this paper are available online. To view these files, please visit the journal online (http://dx.doi.org/10.1136/ bmjopen-2021-053680).

Received 20 May 2021 Accepted 26 November 2021

Check for updates

(c) Author(s) (or their employer(s)) 2021. Re-use permitted under CC BY-NC. No commercial re-use. See rights and permissions. Published by BMJ.

${ }^{1}$ Department of Surgery and Cancer, Imperial College London, London, UK

${ }^{2}$ Department of Infectious Disease Epidemiology, Imperial College London, London, UK

${ }^{3}$ Department of Critical Care, Imperial College Healthcare NHS Trust, London, UK

Correspondence to

Dr Kate Grailey;

k.grailey18@imperial.ac.uk

\section{ABSTRACT}

Objectives This study aimed to investigate the presence of perceived stressors, psychological safety and teamwork in healthcare professionals. As the timeframe for this study spanned the first wave of the COVID-19 pandemic, data were captured demonstrating the impact of the pandemic on these factors.

Design Qualitative interview study.

Setting All staff working within the emergency and critical care departments of one National Health Service Trust in London, UK.

Participants Forty-nine participants were recruited using a purposive sampling technique and interviewed when the first wave of the COVID-19 pandemic had subsided.

Main outcome measures Evaluation of changes in perceived stressors, psychological safety and teamwork in individuals working during the COVID-19 pandemic.

Results The thematic analysis relating to a participant's lived experiences while working during COVID-19 led to the construction of five key themes, including 'psychological effects' and 'changes in team dynamics'. Several psychological effects were described, including the presence of psychological distress and insights into the aetiology of moral injury. There was marked heterogeneity in participants' response to COVID-19, particularly with respect to changes in team dynamics and the perception of a psychologically safe environment. Descriptions of improved team cohesiveness and camaraderie contrasted with stories of new barriers, notably due to the high workload and the impact of personal protective equipment. Building on these themes, a map of key changes arising due to the pandemic was developed, highlighting potential opportunities to provide targeted support.

Conclusions Working on the front line of a pandemic can have significant implications for healthcare workers, putting them at risk of psychological distress and moral injury, as well as affecting team dynamics. There is striking heterogeneity in the manifestation of these challenges. Team leaders can use the themes and qualitative data from this study to help identify areas for management focus and individual and team support.

\section{INTRODUCTION}

The first wave of the COVID-19 pandemic began to have a significant impact on healthcare services in London in March 2020. Healthcare workers were faced with a rapid influx of critically unwell patients, while

\section{Strengths and limitations of this study}

- A strength of this study is the large participant sample, representing a wide range of viewpoints and experiences of those working during the first wave of the COVID-19 pandemic.

By using a qualitative approach, the lived experiences of participants were explored, providing a depth of data and viewpoints that would not typically be captured in quantitative surveys looking for evidence of psychological distress.

- The timing of this study placed the research team in a unique position to capture data related to the COVID-19 pandemic; however, we acknowledge that the initial topic guide was not designed with this in mind.

- A purposive sampling technique was used to recruit participants to this study; however, those interviewed following the first wave of COVID-19 were predominantly weighted to individuals working in critical care.

simultaneously having to create a 'surge' environment to provide the sustained increased capacity required. Healthcare workers were effectively faced with a prolonged major incident, working in hospitals operating above a safe occupancy and intensity for a substantial period of time. ${ }^{1}$ By early April 2021, there had been over 72000 hospitalised patients within London and over 15000 deaths. $^{2}$

Published studies investigating psychological distress in healthcare workers during and after pandemics are predominantly quantitative, using psychological assessment tools such as the Impact of Event Scale ${ }^{3}$ or the Depression, Anxiety and Stress Scale-21. ${ }^{4}$ Psychological distress (typically manifesting as anxiety, depression or post-traumatic stress disorder) has been shown to be prevalent in healthcare workers during and after a pandemic, ${ }^{5-11}$ with studies highlighting a range of contributory factors. There is concordance in the findings of many of these studies-female staff, nursing and younger team members appear to be at highest risk ${ }^{612-15}$; social isolation, fear 
of infection and limited resources all contribute to the development of psychological distress. ${ }^{16}$

A rapid systematic review of 55 studies $^{17}$ (38 of which focused on the COVID-19 pandemic) explored the psychological impact on healthcare workers facing epidemics or pandemics and concluded that fear of the unknown or being infected was paramount. Other prevailing issues include a heightened state of vigilance, ${ }^{18}$ as well as the impact of physical symptoms caused by long hours in personal protective equipment (PPE). Resource rationing, resuscitation decisions and remote communications with relatives are also implicated. ${ }^{19}$ In addition, fears about availability of PPE and mistrust in frequently changing policies have been reported, ${ }^{20-22}$ highlighting the requirement for open and transparent communication. ${ }^{23}$ Working in a pandemic adds an extraordinary level of stress to what is already known to be a stressful working environment, ${ }^{24}$ making the requirement for cohesive teamwork and effective communication even more important. This is particularly challenging in the context of staff redeployment (typically moving staff from non-specialist or outpatient areas to work in high-acuity COVID-19 areas) and the use of PPE. A recognised feature of good teamwork and a positive working environment is the presence of psychological safety, ${ }^{25}$ defined by Khan ${ }^{26}$ in 1990 as 'being able to show and employ one's self without fear of negative consequences of self-image, status or career'. This has been defined within the team setting as an environment 'safe for inter-personal risk taking ${ }^{, 27}$ and is essential for crisis management.

The qualitative interview data presented in this paper tell a story regarding the lived experiences of those working in the first wave of the COVID-19 pandemic, with particular emphasis on changes in perceived stressors, psychological safety, team dynamics and the presence of psychological distress. The original study was a larger mixed methods study designed with the research aim of exploring perceived stressors and the presence of psychological safety in emergency and critical care staff, using a quantitative assessment to explore whether an individual's personality had an influence on this. The study was planned and initiated as part of a behavioural science programme which has been running for some time. It was entirely serendipitous that the study was in place and recruiting as the pandemic evolved. Study recruitment began in September 2019, and as such the period for data collection reflected the first wave of the COVID-19 pandemic in London. The study was paused at the onset of the pandemic; however, we were in a position to resume data collection shortly following this initial surge period, allowing us to provide unique insights into pandemic management across three teaching hospitals within one UK National Health Service (NHS) Trust. It was inevitable that answers to questions in the topic guide evolved to encompass how participants had been affected by COVID-19, and an analytical theme regarding frontline healthcare workers experiences developed.

\section{METHODS}

Non-substantial amendments were made to the study methodology and data collection timeframe as a consequence of disruptions caused by COVID-19 and were approved accordingly.

All staff members working in the emergency departments and critical care departments of three hospitals within one NHS Trust in London were invited to participate. Recruitment to the wider study began in September 2019, with data collection for the part of the study we are presenting in this report representing the immediate postpandemic period. These data reflect activity and personal experience during the first wave and early attempts to return to normal. A purposive sampling technique $^{28}$ was used-participants representing the multidisciplinary team (MDT) with experiences of working in either the critical care or emergency department at all levels of seniority were recruited. This ensured a range of viewpoints reflecting those working in each department were represented in the overall sample.

Participants were provided with written materials prior to their involvement in the study, and once recruited provided written informed consent. Interviews taking place following the enforcement of social distancing and stay at home orders were conducted virtually via the Microsoft Teams platform. The audio was recorded using a handheld audio-recording device and subsequently transcribed with all personal identifying information removed. The topic guide for the semistructured interviews can be viewed in online supplemental file 1 .

Data were continually reviewed throughout the process of data collection using a constant comparative approach.$^{29}$ This was used to inform an iterative approach to the ongoing data collection, influencing subsequent interviews within the boundaries of the topic guide. Participant recruitment ceased when thematic saturation was deemed to have been achieved across all themes constructed during the analysis. ${ }^{30} 31$

Written transcripts were analysed using a thematic analysis technique. An inductive approach was employed, in which the themes identified were strongly linked to the data. The coding process was data-driven, without preconceived ideas or an attempt to fit the data into a pre-existing framework.

This process was facilitated using NVIVO Mac (V.1) software and was performed in line with published guidance on the conduct of a thematic analysis. ${ }^{32}$ The data analysis was a recursive process, moving back and forth within the data while progressing through the sequential stages of a thematic analysis. The first stage involved familiarisation with the data set, in particular the identification of data items that provided information about experiences of working within COVID-19 and how this had influenced team dynamics, perceived stressors and psychological safety. Ongoing review led to the generation of initial codes and included searching for themes across the data set. Through the process of defining and naming these themes, a thematic framework was constructed that 
encompassed participant experiences of working during COVID-19 and how the pandemic had impacted topics explored in relation to the original research questions (namely, perceived stressors, teamwork and psychological safety). We looked for data relating to how these had developed and changed during the first wave of the pandemic. Once these stages were complete the data were rereviewed, with the intention of creating higher order analytical themes.

Discussion between the wider research team took place at all stages of the analysis to confirm the interpretations of the qualitative data and ensure constructed themes accurately reflected the data set. Of the written transcripts, $10 \%$ were coded by a second researcher (AL) and assessed for inter-rater reliability by performing a coding comparison query within NVIVO (V.1) software.

\section{Reflexivity}

$\mathrm{KG}$ is a clinical research fellow with a background in anaesthesia and critical care. AL is a research physiotherapist with a background in respiratory physiotherapy. SB is a clinical academic and consultant in intensive care. Both KG and SB have experience of working during COVID-19 in critical care units. KG and SB have previous experience with the conduct and analysis of qualitative studies in a clinical environment.

The authors were aware of how their own position may affect the study design, analysis and interpretation of the findings, particularly in the context of their own experiences of working during the pandemic. All authors believed that working during the COVID-19 pandemic was likely to impact on perceived stressors and teamwork, although possessed no preconceived ideas regarding the nature of this impact or how this would manifest. The team maintained a reflexive position throughout the analysis to minimise the risk that any presumptions would affect the analysis and interpretation of the study findings.

This manuscript is written in accordance with the Standards for Reporting Qualitative Research, ${ }^{33}$ which can be viewed in online supplemental file 2 . The study protocol is available in online supplemental file 3 .

\section{Patient and public involvement}

No patients or public were involved in the design or implementation of this study.

\section{RESULTS}

Fifty-eight participants were recruited between September 2019 and November 2020 and took part in a semistructured qualitative interview. Forty-nine interviews took place shortly after the first wave of the COVID-19 pandemic had subsided and were conducted virtually via the Microsoft Teams platform. Data from these 49 interviews are presented in this paper.

The duration of these 49 interviews ranged from 12:09 min to $31: 17 \mathrm{~min}$, with an average duration of 20:53 min. Thirty-nine participants in this subgroup were critical care staff, with 10 recruited from the emergency department. This disparity in proportion of participants from each clinical area is a function of those interviewed during the 'post first wave' period. The overall sample is proportional, reflecting the two emergency departments and three critical care departments from which participants were recruited according to a purposive sampling technique. All levels of seniority and the multidisciplinary team are represented in this subgroup of 49 participants, although again there is a smaller proportion of emergency department nurses as the majority of this professional group were recruited and interviewed prior to the onset of the pandemic.

The demographics of participants according to department, profession and level of seniority are displayed in table 1.

Majority of the participants within this subgroup were interviewed in the 3 months immediately following the first wave (19 in July, 6 in August and 10 in September), with the remaining 14 interviews taking place within 6 months (9 in October, 4 in November and 1 in December).

Thematic saturation was defined by the point at which no new codes were added to the coding framework and was achieved both for the total sample and for experiences

Table 1 Demographics of participants according to department, profession and seniority

\begin{tabular}{|c|c|c|c|c|c|}
\hline Clinical department & Participants (n) & Profession & $\begin{array}{l}\text { Participants } \\
\text { (n) }\end{array}$ & Level of seniority & $\begin{array}{l}\text { Participants } \\
\text { (n) }\end{array}$ \\
\hline \multirow[t]{4}{*}{ Emergency medicine } & 10 & Nurse & 2 & Junior & 1 \\
\hline & & & & Senior (band 7 and above) & 1 \\
\hline & & Doctor & 8 & Junior & 6 \\
\hline & & & & Senior (consultant level) & 2 \\
\hline \multirow[t]{6}{*}{ Critical care } & 39 & Nurse & 24 & Junior & 18 \\
\hline & & & & Senior (band 7 and above) & 6 \\
\hline & & Doctor & 9 & Junior & 3 \\
\hline & & & & Senior (consultant level) & 6 \\
\hline & & Physiotherapist & 6 & Junior & 4 \\
\hline & & & & Senior (band 7 and above) & 2 \\
\hline
\end{tabular}




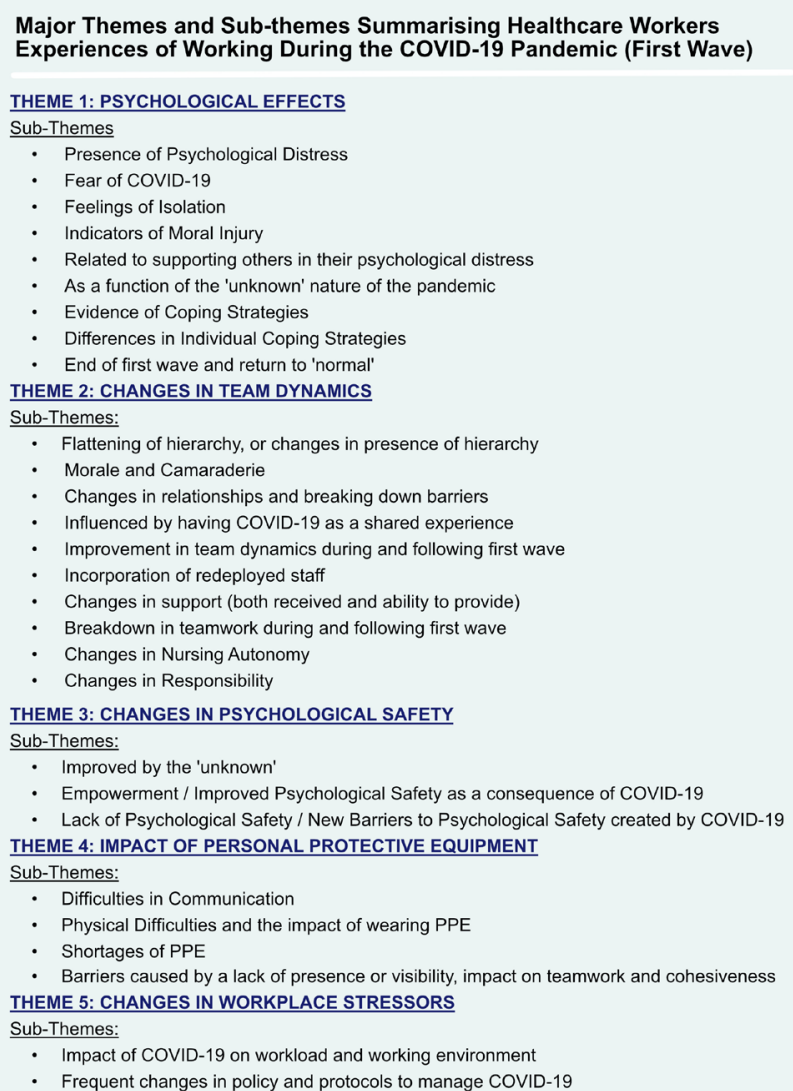

Figure 1 Thematic framework highlighting the major themes and subthemes constructed during the thematic analysis. PPE, personal protective equipment.

related to working during the first wave of the COVID-19 pandemic within the first 40 interviews.

Six of the interview transcripts were selected at random from the interviews taking place after the first wave and were coded by a second researcher (AL). A coding comparison query was performed within NVIVO (V.1). Eighty-eight codes were created during the thematic analysis. Across these the percentage agreement ranged between $92.45 \%$ and $100 \%$. A kappa coefficient of between 0.4 and 0.75 (fair to good agreement) was seen in 14 codes, and $>0.75$ (excellent agreement) in 17 . Discrepancies in coding were resolved through discussion within the research team.

During the constant comparison analysis and subsequent thematic analysis, it became evident that working during the COVID-19 pandemic had made a significant impact on both the individuals and the teams they worked in. Five main themes summarising the lived experiences of participants working on the front line of the COVID-19 pandemic were constructed. This thematic framework is displayed in figure 1 .

The full thematic framework with supporting quotations can be viewed in online supplemental file 4 . Extracts of these data are presented within each theme as figures.

\section{Theme 1: psychological effects}

New psychological distress (defined as a change in emotional status, perceived inability to cope effectively and discomfort ${ }^{34}$ ) arising as a consequence of the pandemic was reported by a high proportion of participants. Participants self-reported feelings of 'burnout' (a phrase used colloquially by many participants, rather than referring to a measured assessment) and mental exhaustion, in addition to low mood, feelings of anxiety and being visibly upset. Participants also described observing psychological distress in their colleagues. Evidence of psychological distress persisted for the duration of the overall data collection, still being described in those interviewed several months after the first wave.

Psychological distress also resulted from fear of COVID-19 itself (either for participants themselves or their family members), with participants feeling vulnerable and anxious about their own risk. There was evidence of psychological distress from participants feeling isolatedeither as an individual (often due to short staffing and high workload) or as a department-struggling to get support from other clinical divisions.

Evidence of moral injury (defined as 'the profound psychological distress resulting from actions which violate an individual's moral or ethical code ${ }^{35-37}$ ) was present as a strong theme within the data set, particularly in those interviewed immediately following the first wave. This was typically related to being unable to complete tasks adequately or provide a standard of care seen as essential prior to the pandemic and appeared to be more prevalent in interviews with more senior staff. Senior staff members also described the psychological impact of supporting other team members, describing a sense of "taking on others" psychological distress.

The 'unknown' nature of the pandemic and COVID-19 was a substantial cause of distress for participants, both in terms of fearing they were unable to adequately treat their patients and due to a lack of prior experience.

There was demonstrable evidence of participants using coping strategies to help manage the impact of the pandemic. These included having a focus on the fact that it would end at some point, acknowledging that not everything could be done in such a scenario or trying to improve the atmosphere and mood of those around them. There were also data to support a subtheme that individuals employed different coping strategies when handling the psychological challenges of the pandemic. Some participants noted that their normal way of interacting with others changed, or that their experience of stress was different from other staff members, reporting being calmer than how they observed others behaving around them.

There was evidence of a change in the psychology of individuals transitioning from working within the first wave of the pandemic to a more 'normal' way of working. There was heterogeneity in the response to this change, with some disappointment at the loss of the new cohesive team structure, a realisation of just how exhausted 


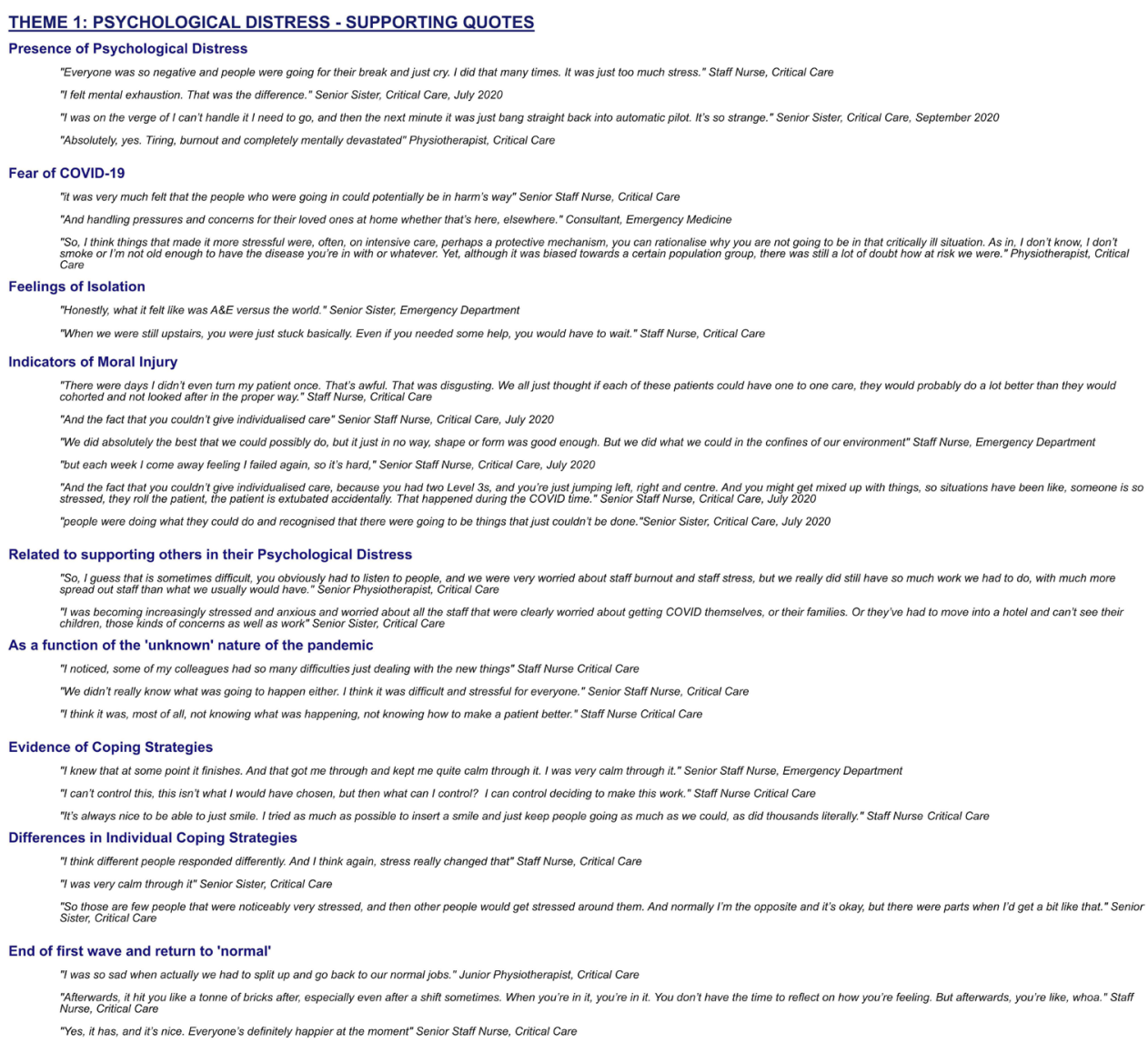

they were and relief that it was over (many of the interviews took place before widespread awareness of the likely possibility of a second wave of COVID-19 in the UK).

Supporting data for this theme can be viewed in figure 2.

\section{Theme 2: changes in team dynamics}

There was marked heterogeneity in participants' experiences when describing the impact of the COVID-19 pandemic on team dynamics. This led to the generation of divergent subthemes encompassing both positive and negative effects. Participants generally reported observing mixed effects on changes in team dynamics, highlighting both positive and negative changes, rather than describing purely positive or negative experiences as a consequence of the pandemic.

Data exploring the presence of a hierarchy suggested that this flattened during the pandemic and was a prominent theme within those interviewed straight after the first wave. This was viewed positively, with the unknown nature of the pathology associated with COVID-19 making it easier to discuss and work openly with more senior members of the team, as those in senior positions did not possess unique insights based on prior experience.

There was a compelling presence of increased staff morale and camaraderie within this data subset, as teams came together to deal with the challenges the pandemic posed. This was particularly represented in those interviewed early in the study timeframe. Traditional working relationships between team members adapted, with staff members becoming closer, developing new modes of communication and stepping into roles outside of their normal job description to help each other.

COVID-19 was a shared experience for all participants, and this commonality created an impression of improved teamwork and cohesiveness. Team members reported feeling closer with an increased ability to rely on and trust each other. Many reported an improvement in team dynamics, related to improved working relationships, increased appreciation of each other and a relaxing of expectations regarding what would normally be achieved, possibly leading to less conflict during handovers.

To manage the rapid increase in workload, many nonspecialist staff were 'redeployed' into the critical care and emergency departments. The impact of this was a pronounced theme within the data, reflecting its significance for pre-existing staff. There were differing feelings regarding the success of incorporating redeployed staff. The addition of redeployed staff created an increased burden for the existing team (frequently due to lack of knowledge and lack of time for training). Not knowing new team members, compounded by face masks and PPE, led to difficulties in communication and effective teamwork. Many participants felt that redeployed staff were not assimilated as well as they could have been-a 
reflection of the workload, time pressures and high stress levels. However, there was also a strong sense of protecting them from questioning by medical staff who might not be aware of their non-specialist status and immense gratitude for the help they provided.

Importantly, negative changes in team dynamics were also reported, with teamwork becoming challenging due to lack of knowledge, a perceived chaotic working environment and as a function of the workload itself. Participants who had seen a positive change in teamwork stated that this had been difficult to maintain once the first wave had ended, mainly due to exhaustion. Later interviews provided participants with more opportunity to reflect on the causes of teamwork breaking down and the attempts made to improve them, such as tools to facilitate communication.

The breadth of participants' experience and roles within the multidisciplinary team allowed the exploration of the impact of the pandemic from numerous viewpoints, providing an appreciation of the multiple challenges and differing responsibilities. Nursing staff described much higher levels of autonomy and decision making, resulting from the redeployment of non-specialist medical staff and reduced specialist staff to patient ratios. Senior medical staff described the challenges of being responsible for multiple clinical areas and an inability to be visible in all areas simultaneously.

The increased responsibility experienced by many participants was associated with mental strain and a risk of psychological distress. The exact manner in which responsibilities changed was dependent on a participant's original job role, but the impact was similar-increased feelings of stress and a sense of being out of control. This change in role also provided further indicators of moral injury-senior staff members reported being unable to support their team members in the way they would normally wish to. Supporting data for this theme can be viewed in figure 3 .

\section{Theme 3: changes in psychological safety}

Psychological safety is defined as 'an environment safe for inter-personal risk taking'. ${ }^{27}$ Participants' perception of the psychological safety of their working environment evolved during COVID-19, with positive and negative changes being reported. The unknown nature of COVID-19 created a more accepting environment in which participants felt they could raise concerns or ideas, with participants feeling less anxious that they might appear to be lacking in knowledge. Psychological safety was also improved by a sense that others understood the potential difficulties an individual might be facing, thereby facilitating open discussion. Many individuals highlighted that the extreme clinical demands precipitated a newly felt 'freedom' to be themselves and speak up. Other antecedents to psychological safety, such as the presence of a hierarchy, explored in other aspects of the thematic framework, are also likely to have influenced changes in psychological safety during the pandemic.
Some new barriers to a psychologically safe environment were described as arising due to the pandemic. These included changes in the team and a hectic working environment, with 'no time' to speak up or propose new ideas. In this context, having 'no time' was a feeling experienced by all those within the working environment, leading to some diminution of psychological safety through a perception that voicing issues would be met with negative responses by those around them due to the recipients' own time constraints. Not having known answers to potential problems or multiple individuals sharing the same concerns were also reported as being detrimental to psychological safety as it created a sense of futility in voicing issues.

Supporting data for this theme can be viewed in figure 4 .

\section{Theme 4: impact of PPE}

The impact of PPE on participants was significant and remained an ongoing issue even after the end of the first wave of the pandemic. Participants described challenges in effective communication, both between individuals in the same location and with team members outside the clinical area. Wearing PPE caused physical distress, hindered identification of other staff members and acted as a barrier to verbal communication. Communication was reported to be hindered both due to the consequences of masks and full coverage hats and gowns covering facial features and difficulty being heard through masks (both in person and via telephone).

Shortages in PPE supply early in the pandemic had a significant effect on participants, leaving individuals concerned for their own safety, as well as restricting individuals from freely entering COVID-19 areas in order to preserve availability.

The impact of PPE corroborates other themes within this data set (notably the presence of feelings of isolation) and was a significant cause of perceived barriers between team members. This eased as supplies in PPE were less restricted, with more team members able to enter the COVID-19 areas.

Those in leadership positions faced significant organisational challenges when trying to mitigate these problems, both in terms of ensuring adequate PPE supply and in creating solutions to improve communication. There were multiple descriptions of cognitive dissonance, as trying to preserve PPE prevented senior staff members from providing visible support in the way they would normally.

Supporting data for this theme can be viewed in figure 5 .

\section{Theme 5: changes in workplace stressors}

Many participants discussed the impact of the pandemic on the presence of the stressors faced within the workplace. An increase in perceived stressors was expressed universally across the participants, irrespective of grade or role within the MDT. A predominant stressor was the 
THEME 2: CHANGES IN TEAM DYNAMICS

\section{POSITIVE CHANGES}

Flattening of hierarchy, or changes in presence of hierarchy

"It's always been pretty easy, but the fact that nobody knew much about it, surely flattened the differences between the senior staff and the junior staff." Staff Nurse Critical Care

"I think that, yes, there definitely was on the ground quite a flattening and a flattening between professions" Staff Nurse Critical Care

"So, I think in that case, it probably did, and I think everybody, regardless of their banding and their grade, were happy to do whatever. So, I think it probably did flatten from that point of view, yes." Senior

Morale and Camaraderie

"But I saw at first-hand what was going on and it was brilliant, they were working more than a team, we were like a family and people just got on with it." Senior Sister, Critical Care

"I think the relationships, generally, was very good. I think, maybe, it's heightened it, if anything, and I think the camaraderie was amazing, between the MDT." Senior Physiotherapist, Critical Care

"I became so much closer with loads of the doctors, the nurses. Whereas usually, people are kind of friends, but less so to the extent that it was during COVID. It was really great, it felt like a little family and I enjoyed coming in to work" Junior Physiotherapist, Critical Care "When it's stressful because of the circumstances that is when people just sort of roll up their sleeves and just get on with it and that's actually quite good in terms of the camaraderie and whatever" Staff Nurse, Critical

Changes in relationships and breaking down barriers

"I became definitely closer with the consultants through COVID than I did before." Senior Sister, Critical Care

"a lot of the doctors would go in and relieve the nurses for their breaks. So when they were coming in to review the patients anyway they would stay there for the hour, and let the ICU nurse step out. And they'd stay

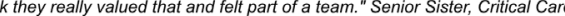

I think I felt part of the bigger hospital, I think we've worked really well together among the specialities, especially at the peak of everything. We were really good, we had systems in place, we had backup systems. Even simple things like if we couldn' get hold of the ITU reg, there was a consultant number, l think it was a direct line to the ward. So, everybody knew the role that they had to play in the bigger picture, so there

Influenced by having COVID-19 as a shared experience

"Yes. Because it's something that you've all been through together, and we learned a lot about each other in that time. Everyone learned about the team a lot." Senior Staff Nurse, Critical Care "We've witnessed something together, but you can say, actually, I can completely depend on that person when everything is really terrible." Senior Sister, Emergency Department "I think there was definitely a feeling of being more united in purpose and in everyone's going through the stress of dealing with this unknown disease." Physiotherapist, Critical Care "but by the same token, other things worked better, simply because we had a common agenda." Consultant, Critical Care

Improvement in team dynamics following first wave

"Or sometimes it can be fantastic, particularly for example, during the pandemic surge, all this suddenly became a much more friendly, and more positive interaction. We all seemed to suddenly become angels, in trying to support

"But yes, definitely some of the normal medical team that were there were incredibly helpful and they valued that a lot." Senior Sister, Critical Care

"Whereas normally the expectation is everything is done when you're handing over, and it can cause an issue or friction if it's not there. And actually that kind of just went." Senior Sister, Critical Car

"we've always been a cohesive team, and I think Covid probably just laid the cement a little bit better." Registrar, Emergency Medicine

\section{CHANGES WITH MIXED EFFECTS}

Incorporation of redeployed staff

"But it was more difficult when I was just surrounded by people whose names I didn't know, whose background I didn't know." Staff Nurse Critical Care

"Many more people we didn't even know the names of, they were coming in and trying to help. But sometimes they didn't receive enough training before coming to intensive care. It was really, really difficult" Staft
Nurse Critical Care

"because also there was so many of them, new people as well, and you were just so grateful that they were there. We were just like thank you. They're not going to be ITU nurses in such a short amount of time with

"But again those new nurses were assigned very sick patients that they probably didn't have the skills to look after yet with machines they didn't understand, and things like that. So the whole thing was just incredibly
stressful" Senior Sister, Critical Care

Changes in Support (both received and ability to provide)

"But generally, I was very well supported by senior [unclear] staff and then, the nursing staff and other health professionals were just amazing. They just got on with it" Junior Doctor, Emergency Department

"it made it tricky if you feel like someone's not coming in." Senior Staff Nurse, Gritical Care

"But from a clinical perspective and for the more junior staff I would say it was less because the consultants just weren't able to go in to the COVID areas because they were also responsible for patients in other areas"
Senior Sister. Critical Care "I think so, yes. I think so. I got a lot of calls asking questions, and I would go in if they needed me, but there were definitely times when we troubleshooted things over the phone, and again that's not something that

"I realised, even myself, I wasn't so supportive to new staff as I could have been because of the workload." Staff Nurse Critical Care

\section{NEGATIVE CHANGES}

\section{Breakdown in teamwork during and following first wave}

"You saw a little bit more separation and slightly less cohesiveness then we would normally see." Consultant, Emergency Medicine

"I'm not sure it was always that rosy, actually, I think that sometimes, the professions were not helping each other" Consultant, Emergency Medicine

"It got worse because everybody had such a huge workload. Nobody really had the chance to support each other." Staff Nurse Critical Care

"actually it's been off the back of the peak that we've seen the tearmwork slightly breaking down just because I think people are mentally and physically exhausted. And so they don't have the time and the energy to be able to put in to do those extra things." Senior Sister, Critical Care

"it's been very difficult, and it is still difficult. So I think the whole teamwork thing has fizzled out a little bit actually." Senior Sister, Critical Care

Changes in Nursing Autonomy

"the nurses on the bed side that were handling the acute situations that you would normally have your reg's come in for" Staff Nurse, Critical Care

"No, normally twice a day we have a doctor's ward round where they are physically on the unit, walk around and review the patients. But that stopped happening. And I suppose it was just the lack of medical presence on the floor, so literally if you had an emergency and you called the emergency call bell, you had to wait for the medical staff to don up, come in whenever" Staff Nurse Critical Care

"It was strange, because you weren't actually working with the team that you're used to, because the ratio changed. So, you'd be the only ITU nurse in the area, and everyone else was redeployed from other areas."

"There wasn't much medical presence, if l'm being honest, on the unit at the time. I think the nurses felt a little bit more like, we're kind of here on our own dealing with it a bit more than in a standard situation because
there's more medical presence." Senior Staff Nurse, Critical Care

Changes in Responsibility

"you could sense that they were under a lot more pressure. Their patient load went up a lot. Their workload doubled." Senior Staff Nurse, Critical Care

"For me, stepping into roles that I wouldn't normally do or things or decision-making I wouldn't normally do, there's a short burst of stress followed by an increase in capability" Staff Nurse Critical Care "But I think the nurses that when they saw me come in they suddenly felt really relieved that I was there, and I just suddenly felt this overwhelming pressure like I don't even know what's going on." Senior Sister, "I think because if something happened with your patients, it was your responsibility to deal with that at the time, because other people there, you can't expect them to know what to do, because they're not trained in

Figure 3 Supporting qualitative data illustrating the theme changes in team dynamics and the associated subthemes (theme 2). 


\section{THEME 3: CHANGES IN PSYCHOLOGICAL SAFETY - SUPPORTING QUOTES}

Improved by the 'unknown'

"Yes. Because no one knew what they were doing. And I like the research so I would be reading journals and I could ask the consultants. We never ventilate anyone APRV, we don't use it. Then I was seeing papers saying that maybe that works for COVID patients. So, you just ask, which was nice to be able to have a more level field on that kind of thing." Senior Staff Nurse, Critical Care

"But there might not be as much experience with just the whole situation, in general. Even if it was things about staff management or I don't know, PPE or positioning or something like that, I do think that it was easier to vocalise, if there was anything" Physiotherapist, Critical Care

Empowerment / Improved Psychological Safety as a consequence of COVID-19

"So, I think there was a facilitated broadening of responsibilities of care and opportunity for discussion. So, I think that was done quite nicely actually thinking about it" Junior Doctor, Critical Care

"I think so. Probably more so after COVID, yes. I think people are a lot more sympathetic I reckon, and more I guess maybe less judged for bringing up issues." Junior Physiotherapist, Critical Care

"It was everyone was just quite candid with each other, and real." Physiotherapist, Critical Care

"People certainly felt very free to contribute in a positive way and did so." Consultant, Critical Care

Lack of Psychological Safety I New Barriers to Psychological Safety created by COVID-19

"I think our structure and hierarchy, above my level, changed during Covid, and sometimes it wasn't always clear who the right person was to go to." Senior Physiotherapist, Critical Care

"But it got to the point where nobody was speaking up because they didn't have time to." Staff Nurse, Critical Care

"I don't think that environment was conducive to anything, to be honest." Consultant, Critical Care

"But it was more difficult when I was just surrounded by people whose names I didn't know, whose background I didn't know. I didn't even know who was more competent than me doing what. If you are with people you don't know much about, then it gets difficult." Staff Nurse Critical Care

Figure 4 Supporting qualitative data illustrating the theme changes in psychological safety and the associated subthemes (theme 3).

hugely increased workload, compounded by the volume of extremely high-acuity patients, changes in the working environment and the requirement to use unfamiliar equipment.

Numerous changes in policies and procedures, often at short notice, were frequently described as being stressors for participants. The nature of how staff were affected by these changes in policy evolved over the course of the interviews.

Those interviewed early in the study described frustrations and challenges with protocols adapting to manage the disease itself and PPE requirements. This evolved as the study progressed to explore the difficulties in navigating new protocols designed to return hospitals to normality, while managing a continuing risk of COVID-19 admissions and areas to nurse these patients.

Supporting data for this theme can be viewed in figure 6 .

The data within the thematic framework were rereviewed to identify potential points during a pandemic response where modifications and strategies could be employed to provide targeted support, reduce the potential impact of new challenges and enhance the positive elements that emerged. Within this there was a focus on the heterogeneity within the data, illustrating how participants within the same environment could respond differently, leading to a variety of consequences. A map of the changes in team dynamics and psychological effects was created, incorporating possible approaches to mitigate negative consequences and promote the positive aspects. This can be viewed in figure 7 .

\section{DISCUSSION}

\section{Statement of principal findings}

Individuals working on the front line during a pandemic are affected in a multitude of ways. Many will suffer psychological distress, moral injury and mental exhaustion. Individuals feel extremely challenged when trying to cope with an increased workload and fear of the disease,

\section{THEME 4: IMPACT OF PERSONAL PROTECTIVE EQUIPMENT - SUPPORTING QUOTES}

Difficulties in communication

"wearing all full PPE so you don't know who everybody is" Senior Staff Nurse, Critical Care

"There's quite a lot of me shouting instructions at people and then PPE communication got difficult, yes, not knowing who anyone was in PPE." Staff Nurse, Critical Care

"I definitely rely on a nurse's name being on the board by the patient's bed or a name tag, and you don't have that. You can't even see their faces. Yes. It definitely makes it a lot harder." Physiotherapist, Critical Care Physical Difficulties and the impact of wearing PPE

"It was wearing PPE for six hours is hard. And, in the beginning, I remember it was half an hour, and we were getting upset. They were all so heavy. And then, by the end of it, wearing it for six hours. And I think it was a bit quiet among all of us. I don't know. It wasn't how we normally would be in the break rooms and things like that." Staff Nurse, Critical Care

"The thing is, the stress isn't even just because of the patient load. It was wearing PPE for six hours is hard" Staff Nurse, Critical Care

Shortages of PPE

"for everybody, and that was one of the biggest problems the whole way through, that you're constantly weighing up, is this reason to leave a room or go into a room worth the impact on PPE?" Staff Nurse Critical

Care

"Yes, definitely, and in fact, the shortage of PPE made that a real problem, because I couldn't just go and do that check-in with multiple people in multiple areas like I normally would do. And I really found that very hard, because actually there just wasn't enough PPE, it wasn't a good use of the available PPE. I didn't mind putting it on and off. If there'd have been more of it, I'd have done it." Staff Nurse Critical Care

"And then if they're working in the PPE, we didn't have the right PPE all the time which was concerning" Staff nurse, Critical Care

Barriers arising as a result of PPE

"Whereas they weren't even coming in to do ward rounds because of COVID it was all done kind of via teams and most of the time without the bedside nurse at all." Staff Nurse, Critical Care

"I didn't see one doctor for at least four days" Senior Staff Nurse, Critical Care

"If anything, there was a massive divide between the nursing team and the medical team. Because the medical team weren't as visible and present on the floor and were doing their rounding from an office, so it infuriated the nursing staff. So there was a massive divide and a lot of animosity. There was a lot of complaints made about that." Staff Nurse, Critical Care "You knew the medical team. They weren't really inside. There was the inside and the outside. It was like through the glass. You're in the PPE and there's everyone outside. So, you're a bit more on your own." Staff
Nurse, Critical Care

Figure 5 Supporting qualitative data illustrating the theme impact of personal protective equipment (PPE) and the associated subthemes (theme 4). 


\section{THEME 5: CHANGES IN WORKPLACE STRESSORS - SUPPORTING QUOTES}

Impact of COVID-19 on workload and working environment

"And then, of course, we've had the pandemic, which is extremely stressful. That was a completely different level of stress that I haven't really been through before." Staff Nurse, Critical Care

"It was madness, absolute madness, but it was life and the tense situation. We adapted to whatever came so it was madness because there was no control about anything." Staff Nurse, Critical Care "The individual patient contact was more stressful because you had a greater awareness of how sick they were and the potential to deteriorate" Consultant, Emergency Medicine

"Pre-pandemic, I normally don't find work that stressful. It was stressful if I had a sick patient, but it would be easier. If there was one sick patient, no other patient would be as sick as them, so everybody would help out. Whereas during COVID, everyone was sick. Everyone was proned and on filter and on every infusion know to man. So, you helped each other but it wasn't like before if that makes sense." Staff Nurse, Critical

"And everything had to be fast paced, but it was just a bit overwhelming." Senior Sister, Critical Care

Frequent changes in policy and protocols to manage COVID-19

"I think the morale is starting to drop a bit because no one can really work out these pathways. "Staff Nurse, Critical Care

"specifically now, given Covid, I think a lot of the stress is a lot of emotional, people's anxiety, meeting expectations, currently having to just be able to adapt all the time, every time there's a new guideline." Registrar, Emergency Medicine

"What was good management this week would probably been seen as not very good management in two weeks' time. That's what, I think, was very stressful and difficult" Consultant, Critical Care

Figure 6 Supporting qualitative data illustrating the theme changes in workplace stressors and the associated subthemes (theme 5).

all while providing support to an increasingly strained team. This is compounded by new barriers created by the pandemic, such as the requirement for PPE.

Beneficial changes in team dynamics also occur as a result of such extreme conditions, including improved camaraderie and enhanced psychological safety, with some individuals finding the experience worthwhile and even enjoyable.

There is a striking heterogeneity in participants' experiences within the data and thematic analysis. This highlights how an individual experience can differ, even when the causative factors and triggers - a pandemic - are the same. Through the phenomenological exploration of lived experiences during the COVID-19 pandemic, we gained an increased understanding of how this may arise. The multiple viewpoints within our sample demonstrate how changes in responsibilities, an individual's job role and experiences will all combine to create different manifestations of how individuals cope with challenges, the psychological distress felt and the subsequent modifications to ways of working.
We provide a unique and indepth narrative regarding an individual's experience and the changes in team dynamics during the first wave of the COVID-19 pandemic. These changes were mapped out, incorporating the likelihood that there will be divergence in these changes depending on the individual. We highlight key areas where targeted interventions can be employed to support healthcare workers and promote beneficial changes in team dynamics.

\section{Strengths and weaknesses of the study}

A strength of this qualitative study is the large participant sample, providing a broad range of viewpoints regarding the impact of a pandemic.

This study was designed to explore perceived stressors, the presence of psychological safety and how personality might influence this. We were uniquely positioned to capture data relating to the impact of COVID-19 on these due to the study timeframe, allowing participants to talk freely about their experiences. However, we recognise the initial topic guide was not designed

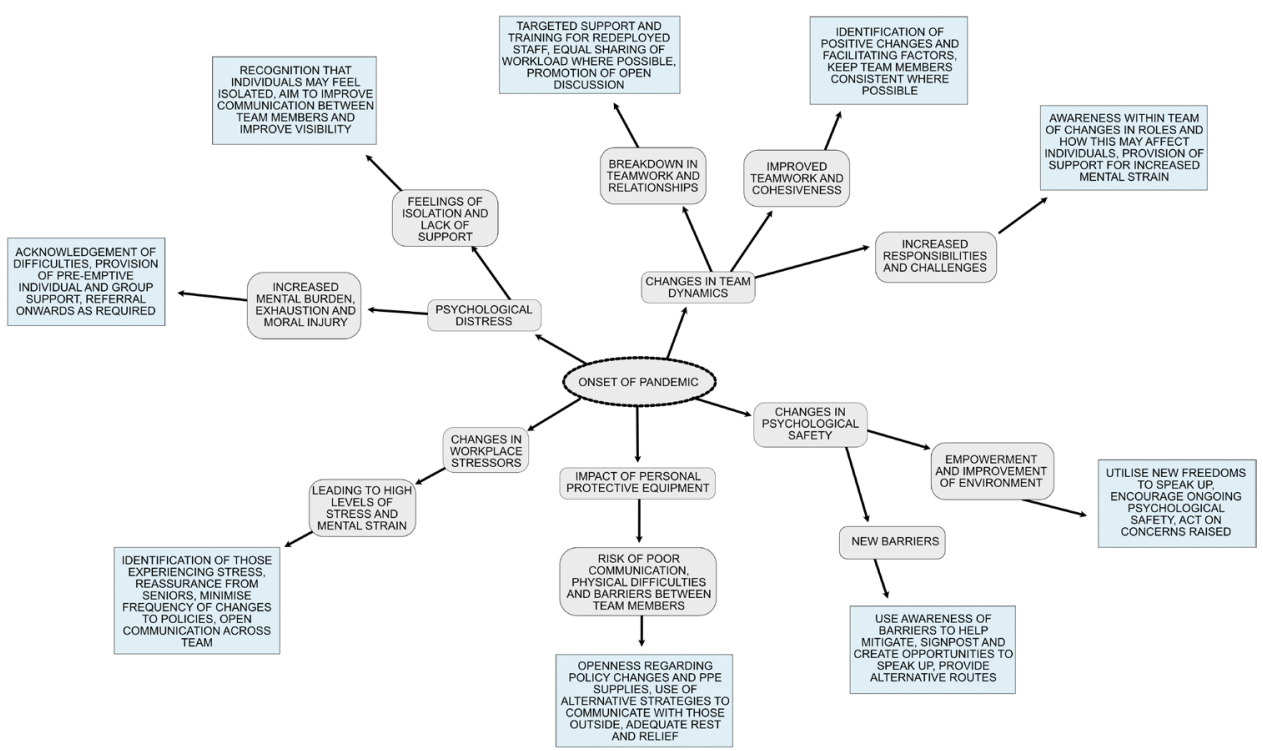

Figure 7 A map of the changes in team dynamics and potential psychological effects as a consequence of the pandemic, with suggestions to mitigate these. PPE, personal protective equipment. 
with the intention of specifically exploring changes in team dynamics and stress as a function of working in a pandemic. A small subtheme where participants explored how they perceived their personality had affected their response to COVID-19 and the development of psychological distress was constructed. However, the majority of the quantitative personality data collected as part of the original study protocol did not relate to the COVID-19 theme and will be analysed and reported separately.

We also acknowledge that while the overall participant sample is purposive, those with experiences relating to COVID-19 are predominantly weighted towards those working in critical care.

\section{Strengths and weakness in relation to other studies}

The qualitative methodology used in this study has allowed the divergent experiences of those working during the COVID-19 pandemic to emerge. This contrasts with many published quantitative studies that use validated tools to obtain a snapshot value regarding the presence of psychological distress. While our qualitative data do not objectively quantify the levels of psychological distress, they distinctively provide the opportunity for positive outcomes as a result of the pandemic to be highlighted (such as camaraderie, improved morale and an increase in psychological safety), telling a broader story than can be achieved with a survey in isolation.

The few existing qualitative studies ${ }^{38-41}$ are small in participant volume and address specific aspects regarding the experiences of healthcare workers during a pandemic. This study, while not designed with COVID-19 in mind, was developed with the broader aims of exploring perceived stressors, teamwork and psychological safety. As such, participants were given the opportunity to discuss their COVID-19 experiences without restriction, creating a rich data set detailing life on the front line of a pandemic.

Our data provide detail on how moral injury can manifest within the participant group, deconstructing some of the causative factors during a pandemic. This topic is not covered in similar studies, although acknowledged to be a significant risk for those working during a pandemic. ${ }^{37} 4243$ We add to these published articles by providing lived examples of how and why this occurred. We also explore how an environment of psychological safety can change and develop during a pandemic.

Acknowledged difficulties in communication and supporting junior team members, challenges in providing training and the presence of high levels of occupational stressors-all identified as being risk factors for the development of negative psychological outcomes ${ }^{44-46}$ are corroborated within this thematic analysis. While not directly asked about, there was no significant theme regarding the benefits or provision of support for mental health during this period present within the qualitative data set. We concur with the conclusion that preventative and proactive measures to support healthcare workers facing high levels of stress associated with working in a pandemic would be beneficial ${ }^{8154748}$ and is underaddressed. ${ }^{49}$

Targets for intervention to support healthcare workers during a pandemic include allaying concerns about health, providing support for staff feeling isolated, ${ }^{50}$ collaborative planning for future outbreaks and the creation of an environment that fosters positive working relationships. ${ }^{51}$ Workplaces should actively encourage mentoring and proactively support those at risk, improve confidence in infection control measures, offer professional support when required and provide recognition of individuals' efforts. ${ }^{11}$ 52-54

\section{Implications for clinicians and policymakers}

The heterogeneity of the data in this thematic analysis regarding participants' experience demonstrates how those in leadership positions should not make any assumptions regarding how individuals will react to a significant challenge, such as a pandemic response. Leaders need to be sensitive to the multiple potential personal responses of their staff, have an expectation that there will be differences in response and plan to have several targeted support systems in place.

An individual's experience of their working day is not only influenced by external stressors, but of their confidence in the team they are working with. A team who knows each other well, has faith in the competence of others and can rely on them for help will begin each shift more confident and prepared for the challenges they may face. ${ }^{55}$ The data in this study highlight how this security was taken away during COVID-19, as individuals had to work in quickly assembled 'teams' based on those available, with strengths and weaknesses unknown. While the presence of these new redeployed team members was viewed positively and a crucial requirement of the pandemic response, it created a new set of barriers and challenges.

The qualitative data in this study will assist those in management and leadership positions with future pandemic preparedness. Our thematic analysis map can be used to recognise how a pandemic can improve team dynamics and encourage psychological safety, allowing leaders to continue to foster this. We have also identified that negative consequences arise and that perceived barriers between teams are often a result of different roles and responsibilities and constraints around activities. These data can be used to provide targeted support-ideally pre-empting the development of significant psychological distress and a breakdown in teamwork. Hospital leaders can use this map to assist in the identification of potential problem areas within their working environments, focusing on the areas that are relevant to them or of greatest need. The experiences of our group of healthcare workers have been used to create potential solutions and improvements to systems and teamwork that can improve the environment and mitigate the risk of psychological distress during crises such as a pandemic. There are also themes and solutions generated within the 
thematic analysis that hospital leaders can explore outside of a pandemic situation (such as the impact of staffing shortages and feelings of lack of support) using our map to identify issues and generate new ways of working.

Although this study was performed in one institution (Imperial College Healthcare NHS Trust), the participants were recruited from three major hospitals, each running 'surged' intensive care services with a variety of subtly differing physical and human environments. While we cannot say with complete certainty that the experiences described and the thematic analysis presented are globally representative, we cannot think of any reason why the issues identified would not have wider resonance in similar health systems operating under epidemic circumstances. We acknowledge that the participants in this study are predominantly weighted towards those working in the critical care environment; however, as the effects of a crisis such as pandemic are wide-reaching and affect many departments within a hospital in similar ways, we would anticipate that the risk and manifestation of psychological distress and other themes developed within this qualitative analysis would affect all staff working in areas affected by the pandemic and not be limited to critical care.

\section{Opportunities for future research}

There are many opportunities for future research. It would be beneficial to design a qualitative study to specifically investigate the incidence of moral injury in participants working during a pandemic and explore the potential psychological consequences of this, both in the short and long term.

There are areas likely to contribute to psychological distress and moral injury during a pandemic response which were not explored within the scope of our topic guide. Further qualitative studies can be designed to evaluate the consequences of resource rationing, virtual communication with relatives and resuscitation decisions.

It would also be valuable to map changes in psychological safety throughout a crisis such as a pandemic using a validated assessment tool $^{27}$ to ascertain whether the perceived changes in psychological safety seen in our qualitative data were reflected in quantitative data. It would also be beneficial to explore some of the themes and solutions generated during the thematic analysis that may be relevant to 'normal' working life (such as low staffing and team morale) to assess the applicability of our analysis to all working scenarios.

\section{CONCLUSIONS}

The data in this qualitative analysis demonstrate how individuals and the teams they work in are impacted by being on the front line of a pandemic in a number of ways, sometimes in contrasting manners. We provide increased detail on the manifestation of psychological distress and moral injury in those working on the front line and highlight how teamwork can be negatively impacted and how the disease process itself can create challenges-both due to the unknown nature of the illness and the PPE required to manage it. Crucially, we highlight that the response to a pandemic is heterogeneous, with some individuals experiencing improved teamwork and psychological safety. The stressors associated with a pandemic response are generally unavoidable, such as the requirement for frequent policy changes and the redeployment of new staff who are unfamiliar with the team and the environment. Our data and analysis show that it is hard to predict how individuals might respond to an event such as a pandemic based simply on characteristics such as job role, seniority or prior experience. As such leaders need to maintain an open mind to their workforce and actively assess the individuals working within each team.

The themes constructed during this analysis can be used by team leaders and managers to mitigate the impact of these stressors and promote the positive consequences. An awareness of the potential for differences in individual staff experience should allow leaders to develop targeted and individual support, with the creation of customised solutions to the difficulties faced.

Contributors $\mathrm{KG}$ and $\mathrm{SB}$ conceptualised and designed this study. $\mathrm{KG}$ and $\mathrm{SB}$ were involved with the recruitment of participants. KG was responsible for data collection, data processing and overall data analysis. AL assisted with data analysis. $\mathrm{KG}, \mathrm{AL}$ and $\mathrm{SB}$ reviewed the data and coding discrepancies in line with qualitative research techniques. KG prepared the original draft manuscript. KG, AL and SB all critically revised the manuscript. All three authors approve the final version of the manuscript. The corresponding author (KG) attests that all listed authors meet the authorship criteria and that no others meeting the criteria have been omitted. SB is the guarantor.

Funding $K G$ received an unrestricted educational grant from Cromwell Hospital. Infrastructure support for this research was provided by the Imperial Comprehensive Biomedical Research Centre (BRC).

Competing interests None declared.

Patient consent for publication Not required.

Ethics approval As per UK Research Governance guidance, studies involving National Health Service (NHS) staff by virtue of their role do not require ethical approval. Health Research Authority approval was granted for this study (reference: 19/HRA/4541) in addition to approval from Imperial College Healthcare NHS Trust's and Imperial College's Joint Research Compliance Office (reference: 19HH5394).

Provenance and peer review Not commissioned; externally peer reviewed.

Data availability statement Data are available upon reasonable request. All data relevant to the study are included in the article or uploaded as supplementary information. All qualitative data are available within the article or uploaded as supplementary information. Further data (deidentified participant data in the form of qualitative interview transcripts and quantitative survey data) are stored in a secure server within Imperial College Healthcare NHS Trust. They are available on reasonable request by emailing the corresponding author.

Supplemental material This content has been supplied by the author(s). It has not been vetted by BMJ Publishing Group Limited (BMJ) and may not have been peer-reviewed. Any opinions or recommendations discussed are solely those of the author(s) and are not endorsed by BMJ. BMJ disclaims all liability and responsibility arising from any reliance placed on the content. Where the content includes any translated material, BMJ does not warrant the accuracy and reliability of the translations (including but not limited to local regulations, clinical guidelines, 
terminology, drug names and drug dosages), and is not responsible for any error and/or omissions arising from translation and adaptation or otherwise.

Open access This is an open access article distributed in accordance with the Creative Commons Attribution Non Commercial (CC BY-NC 4.0) license, which permits others to distribute, remix, adapt, build upon this work non-commercially, and license their derivative works on different terms, provided the original work is properly cited, appropriate credit is given, any changes made indicated, and the use is non-commercial. See: http://creativecommons.org/licenses/by-nc/4.0/.

ORCID iDs

Kate Grailey http://orcid.org/0000-0002-4104-8444

Stephen Brett http://orcid.org/0000-0003-4545-8413

\section{REFERENCES}

1 Mateen BA, Wilde H, Dennis JM, et al. Hospital bed capacity and usage across secondary healthcare providers in England during the first wave of the COVID-19 pandemic: a descriptive analysis. BMJ Open 2021;11:e042945.

2 Healthcare in London. Available: https://coronavirus.data.gov.uk/ details/healthcare?areaType=nhsregion\&areaName=London

3 Horowitz M, Wilner N, Alvarez W. Impact of event scale: a measure of subjective stress. Psychosom Med 1979;41:209-18.

4 Lovibond PF, Lovibond SH. The structure of negative emotional states: comparison of the depression anxiety stress scales (DASS) with the Beck depression and anxiety inventories. Behav Res Ther 1995;33:335-43

5 Ali S, Maguire S, Marks E, et al. Psychological impact of the COVID-19 pandemic on healthcare workers at acute hospital settings in the south-east of ireland: an observational cohort multicentre study. BMJ Open 2020;10:e042930.

6 Tam CWC, Pang EPF, Lam LCW, et al. Severe acute respiratory syndrome (SARS) in Hong Kong in 2003: stress and psychological impact among frontline healthcare workers. Psychol Med 2004;34:1197.

7 Lai J, Ma S, Wang Y, et al. Factors associated with mental health outcomes among health care workers exposed to coronavirus disease 2019. JAMA Netw Open 2020;3:e203976.

8 Kang L, Ma S, Chen M, et al. Impact on mental health and perceptions of psychological care among medical and nursing staff in Wuhan during the 2019 novel coronavirus disease outbreak: a cross-sectional study. Brain Behav Immun 2020;87:11-17.

9 Giusti EM, Pedroli E, D'Aniello GE, et al. The psychological impact of the COVID-19 outbreak on health professionals: a cross-sectional study. Front Psychol 2020;11.

10 Balakumar C, Rait J, Montauban P, et al. COVID-19: are frontline surgical staff ready for this? Br J Surg 2020;107:e195.

11 Muller AE, Hafstad EV, Himmels JPW, et al. The mental health impact of the covid-19 pandemic on healthcare workers, and interventions to help them: a rapid systematic review. Psychiatry Res 2020;293:113441.

12 Pappa S, Ntella V, Giannakas T, et al. Prevalence of depression, anxiety, and insomnia among healthcare workers during the COVID-19 pandemic: a systematic review and meta-analysis. Brain Behav Immun 2020;88:901-7.

13 Si M-Y, Su X-Y, Jiang Y, et al. Psychological impact of COVID-19 on medical care workers in China. Infect Dis Poverty 2020;9:113.

14 Lasalvia A, Bonetto C, Porru S, et al. Psychological impact of COVID-19 pandemic on healthcare workers in a highly burdened area of north-east Italy. Epidemiol Psychiatr Sci 2021;30:e1.

15 Conti C, Fontanesi L, Lanzara R, et al. Fragile heroes. The psychological impact of the COVID-19 pandemic on health-care workers in Italy. PLoS One 2020;15:e0242538.

16 Carmassi C, Foghi C, Dell'Oste V, et al. Ptsd symptoms in healthcare workers facing the three coronavirus outbreaks: what can we expect after the COVID-19 pandemic. Psychiatry Res 2020;292:113312.

17 Cabarkapa S, Nadjidai SE, Murgier J, et al. The psychological impact of COVID-19 and other viral epidemics on frontline healthcare workers and ways to address it: a rapid systematic review. Brain Behav Immun Health 2020;8:100144.

18 Khee KS, Lee LB, Chai OT. The psychological impact of SARS on health care providers. critical care and shock, 2004: 100-6.

19 Mehta S, Machado F, Kwizera A, et al. COVID-19: a heavy Toll on health-care workers. Lancet Respir Med 2021;9:226-8.

20 Azoulay E, Cariou A, Bruneel F, et al. Symptoms of anxiety, depression, and peritraumatic dissociation in critical care clinicians managing patients with COVID-19. A cross-sectional study. Am J Respir Crit Care Med 2020;202:1388-98.
21 Kumar D, Saghir T, Ali G, et al. Psychosocial impact of COVID-19 on healthcare workers at a tertiary care cardiac center of Karachi Pakistan. J Occup Environ Med 2021;63:e59-62.

22 Feingold JH, Peccoralo L, Chan CC, et al. Psychological impact of the COVID-19 pandemic on frontline health care workers during the pandemic surge in New York City. Chronic Stress 2021;5:2470547020977891.

23 Walton M, Murray E, Christian MD. Mental health care for medical staff and affiliated healthcare workers during the COVID-19 pandemic. Eur Heart J Acute Cardiovasc Care 2020;9:241-7.

24 Fiabane E, Giorgi I, Sguazzin C, et al. Work engagement and occupational stress in nurses and other healthcare workers: the role of organisational and personal factors. J Clin Nurs 2013;22:2614-24

25 Rework with Google. Available: https://rework.withgoogle.com/print/ guides/5721312655835136/

26 Khan WA. Psychological conditions of personal engagement and disengagement of work. Acad Manag 1990;33.

27 Edmondson A. Psychological safety and learning behavior in work teams. Adm Sci Q 1999;44:350-83.

28 Robinson RS. Purposive sampling. In: Michalos AC, ed. Encyclopedia of quality of life and well-being research. Dordrecht: Springer Netherlands, 2014: 5243-5.

29 Thorne S. Data analysis in qualitative research. Evid Based Nurs 2000;3:68-70.

30 Ando $\mathrm{H}$, Cousins R, Young $\mathrm{C}$. Achieving saturation in thematic analysis: development and refinement of a codebook. Comprehensive Psychology 2014;3:03.CP.3.4.

31 Lowe A, Norris AC, Farris AJ, et al. Quantifying thematic saturation in qualitative data analysis. Field Methods 2018;30:191-207.

32 Braun V, Clarke V. Using thematic analysis in psychology. Qual Res Psychol 2006;3:77-101.

33 O'Brien BC, Harris IB, Beckman TJ, et al. Standards for reporting qualitative research: a synthesis of recommendations. Acad Med 2014;89:1245-51.

34 Ridner SH. Psychological distress: concept analysis. J Adv Nurs 2004;45:536-45.

35 Litz BT, Stein N, Delaney E, et al. Moral injury and moral repair in war veterans: a preliminary model and intervention strategy. Clin Psychol Rev 2009;29:695-706.

36 Williamson V, Murphy D, Greenberg N. COVID-19 and experiences of moral injury in front-line key workers. Occup Med 2020;70:317-9.

37 Shale S. Moral injury and the COVID-19 pandemic: reframing what it is, who it affects and how care leaders can manage it. BMJ Leader 2020:4:224-7.

38 Shih F-J, Gau M-L, Kao C-C, et al. Dying and caring on the edge: Taiwan's surviving nurses' reflections on taking care of patients with severe acute respiratory syndrome. Appl Nurs Res 2007;20:171-80.

39 Sun N, Wei L, Shi S, et al. A qualitative study on the psychological experience of caregivers of COVID-19 patients. Am J Infect Control 2020;48:592-8.

40 Yin X, Zeng L. A study on the psychological needs of nurses caring for patients with coronavirus disease 2019 from the perspective of the existence, relatedness, and growth theory. Int J Nurs Sci 2020;7:157-60.

41 Schulte EE, Bernstein CA, Cabana MD. Addressing faculty emotional responses during the coronavirus 2019 pandemic. J Pediatr 2020;222:13-14.

42 Greenberg N, Docherty M, Gnanapragasam S, et al. Managing mental health challenges faced by healthcare workers during covid-19 pandemic. BMJ 2020;368:m1211.

43 Čartolovni A, Stolt M, Scott PA, et al. Moral injury in healthcare professionals: a scoping review and discussion. Nurs Ethics 2021;28:590-602.

44 Hong S, Ai M, Xu X, et al. Immediate psychological impact on nurses working at 42 government-designated hospitals during COVID-19 outbreak in China: a cross-sectional study. Nurs Outlook 2021;69:6-12.

45 De Kock JH, Latham HA, Leslie SJ, et al. A rapid review of the impact of COVID-19 on the mental health of healthcare workers: implications for supporting psychological well-being. BMC Public Health 2021;21:1-18.

46 Naushad VA, Bierens JJ, Nishan KP, et al. A systematic review of the impact of disaster on the mental health of medical responders. Prehosp Disaster Med 2019;34:632-43.

47 Moss M, Good VS, Gozal D, et al. A critical care societies collaborative statement: Burnout syndrome in critical care healthcare professionals. A call for action. Am J Respir Crit Care Med 2016;194:106-13.

48 Cai W, Lian B, Song X, et al. A cross-sectional study on mental health among health care workers during the outbreak of corona virus disease 2019. Asian J Psychiatr 2020;51:102111. 
49 Xiang Y-T, Yang Y, Li W, et al. Timely mental health care for the 2019 novel coronavirus outbreak is urgently needed. Lancet Psychiatry 2020;7:228-9.

50 Maunder RG, Lancee WJ, Rourke S, et al. Factors associated with the psychological impact of severe acute respiratory syndrome on nurses and other Hospital workers in Toronto. Psychosom Med 2004;66:938-42.

51 Maunder RG, Lancee WJ, Balderson KE, et al. LongTerm psychological and occupational effects of providing Hospital healthcare during SARS outbreak. Emerg Infect Dis 2006;12:1924-32.

52 Cai H, Tu B, Ma J. Psychological impact and coping strategies of frontline medical staff in Hunan between January and March 2020 during the outbreak of coronavirus disease 2019 (COVID-19) in Hubei, China. Med Sci Monit 2020;26:e924171-1.

53 Ho CS, Chee CY, Ho RC. Mental health strategies to combat the psychological impact of coronavirus disease 2019 (COVID-19) beyond paranoia and panic. Ann Acad Med Singap 2020;49:155-60.

54 Association BM. The mental health and wellbeing of the medical workforce - now and beyond COVID-19, 2020. Available: https:// www.bma.org.uk/media/2475/bma-covid-19-and-nhs-staff-mentalhealth-wellbeing-report-may-2020.pdf

55 D'Lima DM, Murray EJ, Brett SJ. Perceptions of risk and safety in the ICU: a qualitative study of cognitive processes relating to staffing. Crit Care Med 2018;46:60-70. 